\title{
Dislocation loops in overheated free-standing smectic films
}

\author{
A. N. Shalaginov and D. E. Sullivan \\ Department of Physics and Guelph-Waterloo Physics Institute, University of Guelph, Guelph, Ontario N1G 2W1, Canada
}

(November 19, 2018)

\begin{abstract}
Static and dynamic phenomena in overheated free-standing smectic-A films are studied theoretically. The work is based on a generalization, introduced recently by the authors, of de Gennes' theory for a confined presmectic liquid. In this approach, smectic ordering in an overheated film is caused by an intrinsic surface contribution to the film free energy and vanishes at some temperature depending on the number of layers. Here the theory is further generalized to study the dynamics of films with planar inhomogeneities. A static application is to determine the profile of the film meniscus and the meniscus contact angle, the results being compared with those of a recent study employing de Gennes' original theory. The dynamical generalization of the theory is based on a time-dependent Ginzburg-Landau approach. This is used to compare two modes for layer-thinning transitions in overheated free-standing films, namely "uniform thinning" vs. nucleation of dislocation loops. It is concluded that the nucleation mechanism dominates provided there is a sufficiently large pressure difference arising from meniscus curvature. Properties such as the line tension and velocity of a moving dislocation line are evaluated self-consistently by the theory.
\end{abstract}

PACS numbers: 61.30.-v, 61.30.Jf, 64.60.Ht, 64.70.Md, 68.03.Cd, 68.15.+e, 68.60.Dv

\section{INTRODUCTION}

Free-standing films of several smectic-A liquid-crystalline compounds can be heated above the bulk smectic disordering temperature without immediately rupturing, and instead are found to undergo successive layer-by-layer thinning transitions as the temperature is increased [i 6 . The persistence of smectic layering in an overheated thin film is usually attributed to enhanced ordering associated with the free surfaces of the film, as is known to occur in other contexts [7]. There is not yet, however, a clear consensus on the mechanisms by which layer thinning occurs. According to one set of theories [8 11], thinning takes place when the smectic layer structure in the middle of a film vanishes. In an alternative theory [12], supported by experimental studies [13], layer thinning occurs by spontaneous nucleation of dislocation loops prior to the melting of the layer structure in the film interior. This mechanism is not necessarily unrelated to the former, since a sufficient reduction in the degree of interior smectic ordering is required for it to proceed.

One of the key experimental observables is the variation of layer-thinning transition temperatures $T_{c}(N)$ with the number of film layers $N$, which is found to be well fit by the power-law relation $N \propto t^{-\nu}$, where $t=\left(T_{c}(N)-T_{0}\right) / T_{0}$, $\nu \approx 0.70 \pm 0.10$, and $T_{0}$ is close to the bulk transition temperature. Alternative mathematical relations $[10,12,14$, 14 an upper bound 11] for $T_{c}(N)$ have been derived from the different theories. With appropriate fitting parameters, these alternative relations all turn out to agree well with the power-law expression and, thus, are not able to distinguish between the various mechanisms.

In this paper, we examine further the connections between the different proposed mechanisms of layer-thinning transitions. As in several recent works [10, 12, 14], our analysis is based on de Gennes' [15] phenomenological Landau theory for a "presmectic" film of a fluid exhibiting a second-order bulk A-nematic (N) transition. More precisely, we employ a generalization of that theory recently proposed by the present authors [1]. One drawback of de Gennes' original model stems from attributing surface-enhanced smectic ordering to an external-field-like coupling term of constant magnitude, which is more appropriate for a film confined between solid walls. This has the consequence that a weak degree of smectic ordering in a thin film is predicted to persist up to arbitrarily high temperatures. In order to induce layer thinning, recent studies based on this theory have included the effects of a pressure difference $\Delta P$ associated with curvature of the meniscus at the film border 10,14. According to the latter studies, $\Delta P$ must be of a sufficient magnitude to cause layer thinning, although the layer-thinning transition temperatures are found to depend only logarithmically on $\Delta P$. The modified version of de Gennes' theory proposed by the present authors [11] utilizes a different form of the surface contribution to the free energy (suggested by older theories of wetting [16]), which is quadratic rather than linear in the surface order parameter and which restores smectic melting at high temperatures without requiring the pressure term.

According to the theory of Ref. [11], reviewed here in Sec. II, the free energy per area of an overheated smectic film exhibits a discrete sequence of metastable local minima, whose depths decrease with increasing number of layers up to some finite maximum $N_{c r}$ depending on temperature. At or slightly below the temperature for which the 
free-energy well at $N_{c r}$ vanishes, it was assumed in Ref. 11 that the film would spontaneously thin down to a smaller thickness. This assumption makes no statement on "how" layer thinning occurs, which is the question addressed in this paper. We proceed by generalizing the theory to allow for planar inhomogeneities in the film (Sec. III) as well as the dynamical evolution of the film, using a time-dependent Ginzburg-Landau approach (Sec. IV). In the static limit, the theory provides a description of the contact angle between the film and meniscus, which we compare with the recent experimental and theoretical studies of Picano et al. 14]. Using the dynamical theory, we investigate the nucleation of dislocation loops between film domains of different thicknesses and the subsequent growth of the thinner region of the film, which is contrasted with the "uniform-thinning" mechanism for the film to achieve a state of lower free energy. The results are mapped onto the conventional nucleation picture, in which the activation free-energy barrier to nucleation depends on both the difference in well-depths of the homogeneous film regions and the line tension $E$ of the dislocation loop. Here we calculate the line tension $E$, activation energy, and velocity of a growing loop self-consistently in the dynamical model, and evaluate their dependence on temperature and number of layers. It is found that thinning via nucleation of dislocation loops preempts the uniform-thinning mechanism provided the pressure difference $\Delta P$ due to the meniscus is sufficiently large. Conclusions of our findings, and the fact that they are restricted to smectic systems with continuous as opposed to first-order bulk transitions, are discussed in Sec. V.

\section{THEORY OF UNIFORM FILMS}

In this section we review the generalized de Gennes theory in the case of a uniform planar free-standing smectic film: further details can be found in Ref. [11]. The film is modelled by a thin liquid slab bounded by two parallel surfaces located at $z= \pm L / 2$, where $L$ is the film thickness. The degree of smectic order in the film is represented by the complex order parameter $\Psi(z)$, where the real part of $\Psi(z) \exp \left(i q_{0} z\right)$ describes spatial modulation of the density. Here $q_{0}=2 \pi / d$, with $d$ being the unstressed smectic layer spacing. The order parameter is parameterized as

$$
\Psi(z)=\psi(z) \exp [-i \phi(z)]
$$

where $\psi(z)$ is the amplitude and $\phi(z) \equiv q_{0} u(z)$ is a phase proportional to the layer displacement $u(z)$.

The Landau free energy per unit area of the film [17] is taken to be

$$
\begin{aligned}
f & =\frac{1}{2} \int_{-L / 2}^{L / 2} d z\left[r \psi^{2}+\frac{1}{2} g \psi^{4}+C\left(\nabla_{z} \psi\right)^{2}+C \psi^{2}\left(\nabla_{z} \phi\right)^{2}\right] \\
& +\frac{1}{2} r_{s}\left[\psi^{2}(L / 2)+\psi^{2}(-L / 2)\right]-h_{s}[\psi(L / 2)+\psi(-L / 2)],
\end{aligned}
$$

where $C$ is an elastic constant. This model free energy generalizes that used in Refs. 10,12, 14,15, by including both a quartic term $(g / 4) \psi^{4}$ with $g>0$ in the bulk free-energy density and quadratic contact terms $r_{s} \psi^{2}( \pm L / 2) / 2$ [16] in the contribution of the surface layers. As in those works, the bulk free-energy density employed in Eq. (2) is strictly applicable only to a system exhibiting a second-order bulk smectic disordering transition, which occurs at $r=0$ in this mean-field theory. We express $r=a\left(T-T^{*}\right)$, where $T^{*}$ denotes the bulk mean-field transition temperature [19]. Euler-Lagrange equations determining $\psi(z)$ and $\phi(z)$ are obtained by functional minimization of Eq. (2)).

Two alternative versions of this theory for surface-enhanced ordering of smectic free-standing films have been investigated. In the first approach [10,12, 14, following the original work of de Gennes [15], such ordering is induced solely by the surface field $h_{s}$, with $r_{s}=0$. These studies have also set $g=0$. Refining some steps of these earlier analyses, which were restricted to the asymptotic limit $L \gg \xi$, we find that the equilibrium free energy of this model is given by [20]

$$
f(L)=-h_{s} \psi(L / 2)=-\frac{h_{s}^{2} \xi}{C} \frac{\sinh (L / \xi)}{\left[\cosh (L / \xi)-\cos \left(q_{0} L\right)\right]},
$$

where $\xi \equiv \sqrt{C / r}$ is the bulk correlation length. Note that Eq. (3) diverges on approaching the bulk critical temperature, as does the order parameter $\psi(z)$ throughout the film, a consequence of neglecting the non-harmonic term $g \psi^{4}$ in Eq. (2). This feature has been mitigated in some versions of the theory 10,14,21 by the somewhat ad-hoc procedure of fixing $\psi(L / 2)$ instead of $h_{s}$. It is also worthwhile to note that the minima of Eq. (3) do not occur precisely at the values $L=N d$, but at slightly compressed values $L<N d$ of the film thickness, which is due to an interplay between finite-size and surface-ordering effects. This feature also holds for the more general model in Eq. (2). 
In Ref. [11], smectic ordering in an overheated film is attributed to a non-zero value $r_{s}<0$, with $h_{s}=0$. In the following we refer to this as the $r_{s}$-model. In this case, the Euler-Lagrange equations obtained from Eq. (2) always admit a trivial solution $\psi(z)=0$ describing a disordered state of the film, which is the only solution at sufficiently high temperature. The numerical solution of those equations, which consider $g \neq 0$, is described in Ref. [11]. The variation of the equilibrium free energy $f(L)$ typical for this model is depicted in Fig. 1. The free energy exhibits a set of wells with centers situated approximately at $L=N d$ and depths diminishing with $N$, while $f(L)$ vanishes over non-zero ranges of $L$ between the wells. Although not discernable in the figure, the slope of the free energy smoothly approaches zero at the limits where $f(L) \rightarrow 0$. In the temperature range $T<T_{s}$, where $T_{s}=T^{*}+r_{s}^{2} /(C a), f(N d)$ for any $N$ is less than some negative threshold depending on $T$. For temperatures $T>T_{s}$, wells with non-zero depths occur only up to a finite number $N_{c r}$. This in the case in Fig. 1], where $N_{c r}=11$. It was argued in Ref. [11] that the temperature at which the free-energy well for $L=N_{c r} d$ disappears, which we will call the "maximum temperature" for an $N_{c r}$-layer film, is an upper limit for the layer-thinning transition temperature $T_{c}\left(N_{c r}\right)$. Films of all $N<N_{c r}$ can still exist as metastable states and, in principle, thinning could then occur to any one of these states.

We note the following scaling of the $r_{s}$-model free energy, used in Fig. 1 and in subsequent analysis. On expressing distances in units of the layer-spacing $d$, one sees that the free energy in Eq. (2) can be expressed as $f\left(L, r, g, r_{s}, C\right)=$ $(C / d) \hat{f}\left(L / d, \hat{r}, \hat{g}, \hat{r}_{s}\right)$, where $\hat{r}=r d^{2} / C, \hat{g}=g d^{2} / C$, and $\hat{r}_{s}=r_{s} d / C$. In principle, the parameter $\hat{g}$ could also be pulled out from $\hat{f}$ by suitable scaling of the order parameter $\psi$, but we have found it convenient for numerical analysis to set this at the (arbitrary) small value $\hat{g}=0.01$ and leave the scaling of $f$ in the form indicated.

\section{NONUNIFORM FILMS: STATICS}

In order to study dislocation loops and associated phenomena in free-standing films, we need to generalize the theory of Sec. II to incorporate planar inhomogeneities in a film. This could be carried out by generalizing the free energy of Eq. (2) to include horizontal gradients of the order parameter $\Psi$ and nematic director, consistent with de Gennes' more general theory of the N-A transition [22], as well as of the film thickness $L$. Full analysis of this theory would require minimizing the resulting free energy with respect to $\Psi$ and the director. In the remainder of this paper we will examine a simplified phenomenological description using an interface-displacement theory [23], in which the free energy is expanded in gradients of $L$. To leading order in these gradients, the film free energy $F$ is given by

$$
F=\int d^{2} r_{\perp}\left[f(L)+\frac{1}{2} D\left(\nabla_{\perp} L\right)^{2}\right] .
$$

Here the horizontal direction is represented by $\mathbf{r}_{\perp}$, with Cartesian components $(x, y), \nabla_{\perp}=\left(\nabla_{x}, \nabla_{y}\right), L=L\left(\mathbf{r}_{\perp}\right)$ is the spatially-varying film thickness, and $f(L)$ is the equilibrium free energy per area of a film of uniform thickness $L$, obtained from Eq. (2). The coefficient $D$ characterizes the "stiffness" [23] of the film surfaces, which in principle could be derived by analysis of the underlying de Gennes theory. We expect that $D \approx \gamma / 2$, where $\gamma$ is the liquid-vapor interfacial tension. Generally $D$ could vary with $L$ due to changes in the degree of smectic ordering, but such changes are expected to be weak and here we will assume that $D$ is constant.

An additional term may enter Eq. (4) due to the existence of a positive pressure difference $\Delta P=P_{\text {air }}-P_{\text {liquid }}$ across the surface of the meniscus surrounding the film. Such a pressure difference produces a shift $\Delta \mu$ in the chemical potential of the film molecules from their value at coexistence with the vapor phase across a planar interface [24, 25]. This leads to the replacement of $f(L)$ in Eq. (4) by $\tilde{f}(L)$, where

$$
\tilde{f}(L)=f(L)+\Delta P L .
$$

The main effect of the $\Delta P$ term is to shift the depths and to a slight extent the positions of the smectic minima of the effective free energy $\tilde{f}(L)$ with respect to those of $f(L)$, possibly eliminating minima occurring at large $L$ [10].

The static free energy Eq. (位) can be used to obtain the shape of the meniscus at the edge of a free-standing film, following the analysis of Ref. [14] based on the original de Gennes model. Related considerations have been applied to describe the shape of liquid droplets on a solid substrate [26,27]. The profile of the meniscus is found by minimizing Eq. (雨) with respect to $L$ (replacing $f$ by $\tilde{f}$ ), subject to the boundary condition that the main part of the film has a thickness $L \equiv H \approx N d$. Assuming that $L$ varies only in the $x$-direction, the resulting Euler-Lagrange equation is

$$
D \frac{d^{2} L}{d x^{2}}-\frac{\partial \tilde{f}}{\partial L}=0
$$

The first integral of this equation, with the boundary condition $L(-\infty)=H$, is 


$$
\frac{D}{2}\left(\frac{d L}{d x}\right)^{2}=\tilde{f}(L)-\tilde{f}(H)
$$

which has the implicit solution

$$
x=\sqrt{\frac{D}{2}} \int_{L(0)}^{L(x)} d L^{\prime}\left[\tilde{f}\left(L^{\prime}\right)-\tilde{f}(H)\right]^{-1 / 2} .
$$

Here the origin $x=0$ and corresponding value $L(0)>H$ are arbitrary. Note that no boundary conditions need to be specified at large positive $x$, approaching the meniscus border. This reflects the fact that those boundary conditions are non-universal and depend on how the film is created [28]. However, the description based on the above equations is only valid for small $\nabla_{\perp} L$; deep in the meniscus, higher-order terms in $\nabla_{\perp} L$ should enter [14,28].

Figure 2 shows the solutions to Eq. (8) for the $r_{s}$-model using the same parameters as in Fig. 1. Two cases are shown, the solid line corresponding to $\Delta P=0$ while the dotted line corresponds to $\Delta P=0.05 C / d^{2}$. (The reduced unit for $\Delta P$ is consistent with that for $f$ discussed at the end of Sec. 1 . Following from this scaling and Eq. (8), the horizontal distance $x$ is expressed in units of $R_{s c} \equiv d \sqrt{D d / C}$.) In both cases, the meniscus profile is fairly smooth. At low temperatures, when the smectic-A phase is stable in bulk, the meniscus is expected [25] to consist of a set of edge dislocations which change the film thickness by steps of height $\approx d$. The absence of such distinct steps in Fig. 2 reflects the fact that here we are in the overheated regime.

For $T<T_{s}$, the function $f(L)$ tends to a non-zero value $f(\infty)$ with increasing $L$. The latter represents the contribution of surface-induced smectic ordering to twice the interfacial tension $\gamma$ of the liquid-vapor interface of a semiinfinite liquid. Neglecting small oscillations of $f(L)$ about $f(\infty)$ and defining the meniscus slope angle $2 \theta \approx d L / d x$, Eq. (7) leads to

$$
\theta^{2}=\frac{1}{2 D}[f(\infty)-f(H)+\Delta P(L-H)]
$$

On assuming that $D=\gamma / 2$ and extrapolating the function in Eq. (9) down to $L=H$, we obtain for the contact angle $\theta_{m}$ between the meniscus and film,

$$
\theta_{m}^{2}=\frac{1}{\gamma}[f(\infty)-f(H)]
$$

independent of $\Delta P$. This result agrees with that derived in Ref. [14]; we note that essentially equivalent relations were derived some time ago in the case of soap films [24,29]. Note also that Eq. (9) predicts growth of $\theta$ with increasing $L$. The meniscus curvature $\kappa$ is found to be

$$
\kappa \equiv \frac{d \theta}{d x}=\frac{d \theta}{d L} \frac{d L}{d x}=\frac{d \theta^{2}}{d L}=\frac{\Delta P}{\gamma}
$$

which is just the Laplace law.

Using the original de Gennes model free energy, Eq. (3), one finds that the contact angle diverges as $T$ approaches $T^{*}$ from above, whereas the experimental results of Ref. [14] indicate regular behavior in this region. This feature was alleviated in the calculations of Ref. [14], based on the de Gennes model, by fixing $\psi(L / 2)$ instead of $h_{s}$. Although this eliminates the divergence of the contact angle in the vicinity of the bulk transition point, the resulting model predicts a different anomaly, namely that $\theta_{m}$ vanishes as $T \rightarrow T^{*}$ for all $N$. The divergence of the contact angle near the bulk second-order transition in the original de Gennes model can be removed by setting $g \neq 0$. Here we present results for $\theta_{m}$ using the $r_{s}$-model, although qualitatively similar results are obtained using $r_{s}=0$ with $h_{s} \neq 0$ and $g \neq 0$. Figure 3 shows $\theta_{m}^{2}$, in units of $C /(d \gamma)$, as a function of $\hat{r} \propto\left(T-T^{*}\right)$ for various number of layers $N$, using the free energy $f(L)$ depicted in Fig. 1. It is seen that $\theta_{m}$ remains non-zero on approaching the bulk transition temperature and increases with decreasing $N$, in agreement with experiment [14]. We also find that the contact angle vanishes above the maximum temperature for a given number of layers. This is in contrast with the model of Ref. [14], which yields a small but non-zero contact angle for arbitrarily large temperature and film thickness. However, as seen in Fig. 3, the maximum in $\theta_{m}$ as a function of temperature is fairly insensitive to the number of layers, which does not accord with the experimental results.

Comparison of Fig. 3 with the experimental data [14] for $\theta_{m}^{2}$ indicates that the scaling unit $C /(d \gamma)$ should be on the order of $10^{-2}$. Taking $D=\gamma / 2$, we then estimate the in-plane distance scale unit to be $R_{s c} \equiv d \sqrt{D d / C} \approx$ $(10 / \sqrt{2}) d \approx 2 \times 10^{-8} \mathrm{~m}$, where we have used the value $d=3 \times 10^{-9} \mathrm{~m}$ 30]. 


\section{DYNAMICS OF NONUNIFORM FILMS}

\section{A. Time-dependent Ginzburg-Landau equation}

To study the dynamics of a thinning free-standing smectic film, we consider the latter to be a two-dimensional object with a free energy characterized by its thickness $L$, given by Eq. (4) with, generally, $f$ replaced by $\tilde{f}$. We will focus on dynamical processes with large enough characteristic times to neglect inertial effects. Although the details of relaxation are undoubtedly quite complicated, here we will proceed by assuming the simplest possible dissipative dynamics for $L$, based on a time-dependent Ginzburg-Landau (TDGL) equation [31]. This equation is appropriate for describing the dynamics of a non-conserved variable, which $L$ can be regarded in the case of a film open to the exchange of molecules with the meniscus. The TDGL equation is

$$
\eta \frac{\partial L}{\partial t}=-\frac{\delta F}{\delta L}=\left[D \nabla_{\perp}^{2} L-\frac{\partial \tilde{f}}{\partial L}\right] .
$$

Here $\delta F / \delta L$ is the functional derivative of $F$, giving the thermodynamic force which drives the system toward equilibrium, $t$ is time and $\eta$ is a kinetic coefficient which we will assume to be constant.

Two opposing mechanisms for thinning of an overheated smectic film are "uniform thinning" (i.e., with $\nabla_{\perp} L=0$ ) and via nucleation of dislocation loops. If the initial film thickness $L_{0} \approx N d$ is uniform and at a local minimum of the shifted free energy $\tilde{f}$, then it will remain so indefinitely according to Eq. (12). (This picture neglects possible disruption due to thermal fluctuations, which we neglect in this work.) Under small displacements of the thickness from the initial value $L_{0}$, the film will be restored to that initial thickness. Hence uniform thinning can only occur at the maximum temperature of an $N$-layer film, and then only if $\Delta P>0$ [32].

To examine the growth of dislocation loops, for simplicity we first consider a one-dimensional solution of Eq. (12), in the form of an infinite straight-line kink separating domains of thicknesses $L_{1}$ and $L_{2}$ and moving along the $x$-axis:

$$
L=\Phi\left(x-v_{\infty} t\right)
$$

where the function $\Phi$ and kink velocity $v_{\infty}$ are to be determined. Substituting Eq. (13) into Eq. (12) yields the ordinary differential equation

$$
D \Phi^{\prime \prime}+\eta v_{\infty} \Phi^{\prime}-\frac{\partial \tilde{f}(\Phi)}{\partial \Phi}=0
$$

with boundary conditions

$$
\begin{aligned}
\Phi(-\infty) & =L_{1}, \\
\Phi(\infty) & =L_{2},
\end{aligned}
$$

where the prime symbols ( $\prime)$ denote derivatives of $\Phi$ with respect to its argument. The thicknesses $L_{1}$ and $L_{2}$ are at local minima of $\tilde{f}(L)$. Usually we will take these to be adjacent minima, with $L_{1} \approx(N-1) d, L_{2} \approx N d$, and $\tilde{f}\left(L_{1}\right)<\tilde{f}\left(L_{2}\right)$.

Equation (14) has a well-known mechanical analogy [33,34. It can be considered as the dynamical equation describing movement of a particle of "mass" $D$ in a medium with "friction coefficient" $\eta v_{\infty}$ and subject to a "potential energy" $-\tilde{f}(L)$. Note that there is no stationary solution $\left(v_{\infty}=0\right)$ of this equation unless the depths of the minima of $\tilde{f}(L)$ are equal. For any function $\tilde{f}(L)$ characterized by two unequal adjacent minima, as is the case here, there should be a unique solution for the velocity $v_{\infty}>0$ and function $\Phi$ describing the profile of the kink, which moves toward the region of thickness $L_{2}$ in order to eliminate the domain of higher free energy. Under conditions where the higher minimum of $\tilde{f}(L)$ at $L_{2}$ vanishes and becomes a point of zero curvature, as happens in the present model at the maximum temperatures, the velocity $v_{\infty}$ and kink shape may become non-unique. In other contexts [33,35,36], this is called a state of marginal stability. Here, we always find (Sec. IV C) that uniform thinning occurs under these conditions when $\Delta P>0[32]$.

The solutions of the one-dimensional equation Eq. (14) turn out to be relevant in the more general two-dimensional case, as discussed in the next subsection. 


\section{B. Nucleation of dislocation loops}

If a dislocation loop separating $N$ - and $(N-1)$-layer regions is nucleated, initially it will be of finite size. According to the conventional nucleation picture (see, e.g., Refs. [12,22,28] for the case of smectic films), the loop then will either expand or collapse depending on whether its initial radius is greater or smaller than some "critical" value. Here we are interested in determining the critical radius, the associated activation free energy, and the subsequent dynamical evolution of the loop.

We assume that the dislocation loop is a circle. Using in-plane polar coordinates, with origin at the center of the loop and radial distance denoted $r$, Eq. (12) becomes

$$
\eta \frac{\partial L}{\partial t}=\left[D \frac{1}{r} \frac{\partial}{\partial r}\left(r \frac{\partial L}{\partial r}\right)-\frac{\partial \tilde{f}}{\partial L}\right] .
$$

The associated boundary conditions are

$$
\begin{gathered}
\left(\frac{\partial L(r)}{\partial r}\right)_{r=0}=0, \\
L(\infty)=L_{2} \approx N d .
\end{gathered}
$$

The film thickness in the center of the loop at $r=0$ will usually be close to the value $L_{1} \approx(N-1) d$. The change in free energy due to formation of the loop is

$$
\begin{aligned}
\Delta F & =\frac{1}{2} \int d^{2} r_{\perp}\left[2 \tilde{f}(L)-2 \tilde{f}\left(L_{2}\right)+D\left(\nabla_{\perp} L\right)^{2}\right] \\
& =\pi \int_{0}^{\infty} d r r\left[2 \tilde{f}(L)-2 \tilde{f}\left(L_{2}\right)+D\left(\frac{\partial L}{\partial r}\right)^{2}\right] .
\end{aligned}
$$

In the case of a stationary solution of Eq. (16), corresponding to a "critical" nucleus, $\Delta F$ is the activation free energy.

The profile of $L(r)$ describing a dislocation loop should have a kink-like structure, with $\partial L / \partial r \approx 0$ everywhere except within a narrow region centered around some value $r=R$. Numerical solution of the stationary limit of Eq. (16), with $(\partial L / \partial t)=0$ and the boundary conditions Eq. (17) is difficult, precisely because this is associated with a unique "critical" value of the loop radius $R$. We have found it more expedient, and of more general relevance, to solve the full time-dependent partial differential equation Eq. (16), using a standard subroutine (NAG Fortran D03PCF). Numerical analysis of the differential equation, starting from initial trial profiles mimicking the expected kink structure, shows that solutions in the form of moving kinks are, indeed, dynamically stable. Hence it is worthwhile to consider from the outset a solution to Eq. (16) in the form of a moving kink:

$$
L(r, t)=\Phi(r-R(t)) .
$$

As discussed some time ago in a general context by Chan [37, this form is not an exact solution of Eq. (16) but should be a good approximation if the kink radius $R$ is much larger than its width denoted $\Delta R$. Using Eq. (19) in Eq. (16) and approximating $1 / r$ by $1 / R$, we arrive again at Eq. (14), but with $v_{\infty}$ defined as [37]

$$
v_{\infty}=\frac{d R}{d t}+\frac{D}{\eta} \frac{1}{R}
$$

This transforms the two-dimensional problem to the one-dimensional case, which requires finding the pair $\left(\Phi, v_{\infty}\right)$ with constant $v_{\infty}$, satisfying Eq. (14) with boundary conditions Eqs. (15). The quantity $v_{\infty}$ is the asymptotic velocity of a loop with sufficiently large radius $R$ and depends (for given $D$ and $\eta$ ) only on the function $\tilde{f}(L)$ and its chosen pair of minima. Our numerical analyses of the original two-dimensional equation Eq. (16) show that both the kink shape $\Phi$ in the moving coordinate frame and the right-hand-side of Eq. (20) remain practically constant as the kink moves. Thus, while the results to be reported in Sec. IV the one-dimensional mapping based on Eqs. (14), (19) and (20) usually is an excellent approximation and provides, as discussed below, a useful framework for interpreting the results.

Note that setting the time derivative of $R$ equal to zero in Eq. (20) yields an expression for the critical loop radius,

$$
R_{c}=\frac{D}{\eta v_{\infty}}
$$


If $v_{\infty}$ is known, then $R(t)$ at arbitrary time can be found by solving Eq. (20) [37], which gives the relation

$$
\begin{aligned}
t-t_{0} & =\frac{1}{v_{\infty}} \int_{R_{0}}^{R} d R^{\prime} \frac{R^{\prime}}{R^{\prime}-R_{c}} \\
& =\frac{1}{v_{\infty}}\left(R-R_{0}+R_{c} \ln \left|\frac{R-R_{c}}{R_{0}-R_{c}}\right|\right),
\end{aligned}
$$

where $R_{0}$ is the radius at an arbitrary initial time $t_{0}$.

In conventional treatments of nucleation phenomena, properties of the critical nucleus such as $R_{c}$ are expressed in terms of purely thermodynamic quantities, and it is worthwhile to show that connection in the present context. From Eq. (14), one finds that $\Phi$ and $v_{\infty}$ satisfy

$$
\frac{d}{d X}\left[\frac{1}{2} D\left(\Phi^{\prime}\right)^{2}-\tilde{f}(\Phi)\right]=-\eta v_{\infty}\left(\Phi^{\prime}\right)^{2},
$$

where $X \equiv x-v_{\infty} t$ is the argument of $\Phi$. Integrating Eq. (23) over $X$ using the boundary conditions Eq. (15) yields

$$
v_{\infty}=\frac{D}{\eta E}\left[\tilde{f}\left(L_{2}\right)-\tilde{f}\left(L_{1}\right)\right]
$$

where the quantity $E$, which will be identified as the line tension of the dislocation loop, is defined as

$$
E \equiv D \int_{-\infty}^{\infty} d X\left(\Phi^{\prime}\right)^{2}=D \int_{L_{1}}^{L_{2}} d \Phi \Phi^{\prime}
$$

Consistent with the one-dimensional mapping, the lower limit of integration over $X$ has been set to $-\infty$. To a first approximation, Eq. (24) shows that $v_{\infty}$ is proportional to the free energy difference $\tilde{f}\left(L_{2}\right)-\tilde{f}\left(L_{1}\right)$ and, hence, vanishes if that difference is zero. Using Eq. (24), Eq. (21) becomes

$$
R_{c}=\frac{E}{\tilde{f}\left(L_{2}\right)-\tilde{f}\left(L_{1}\right)} .
$$

One can show that Eqs. (25) and (26) for $E$ and $R_{c}$ are consistent with standard arguments of nucleation theory. The free energy change Eq. (18), with the solution for $L$ in the form of Eq. (19), can be approximated as

$$
\Delta F=-\pi R^{2}\left[\tilde{f}\left(L_{2}\right)-\tilde{f}\left(L_{1}\right)\right]+2 \pi R E .
$$

This follows on approximating the integrand in Eq. (18) by the constant $\tilde{f}\left(L_{1}\right)-\tilde{f}\left(L_{2}\right)$ inside a circle of radius $R-\Delta R / 2$, neglecting the integrand for $r>R+\Delta R / 2$, and using Eq. (23) within the kink region of width $\Delta R$. Equation (27), with $E$ given by Eq. (25), is then obtained on assuming $\Delta R \ll R$. The first term in Eq. (27) is the decrease in film free energy due to the difference $\tilde{f}\left(L_{2}\right)-\tilde{f}\left(L_{1}\right)$ [38] while the second term is the free energy which has to be overcome due to the line tension of the loop. Maximizing $\Delta F$ with respect to $R$ yields the critical radius $R_{c}$ given by Eq. (26). The corresponding activation free energy $F_{a c t}=\Delta F\left(R_{c}\right)$ is

$$
F_{a c t}=\frac{\pi E^{2}}{\tilde{f}\left(L_{2}\right)-\tilde{f}\left(L_{1}\right)}=\pi R_{c} E .
$$

The expression for $E$ in Eq. (25) agrees with a familar mean-field relation for the tension of a stationary interface in terms of its profile shape 19. Here that relation also applies to a moving kink of sufficiently large radius, under the assumption (supported by our numerical studies) that the profile shape is preserved during its motion.

One final point to note concerns the physical interpretation of the equation of motion Eq. (20) for $R(t)$. That equation is equivalent to the balance of thermodynamic and dissipative forces per unit length of the dislocation line:

$$
\frac{1}{2 \pi R}\left(\frac{d \Delta F}{d R}\right)+\eta \frac{E}{D}\left(\frac{d R}{d t}\right)=0
$$

This yields Eq. (20) on using Eqs. (24) and (27), and agrees with the model of dislocation-loop dynamics described by Geminard et al. [28], on identifying the mobility $\mu$ used by these authors with the quantity $D d /(\eta E)$. The study of dislocation-loop dynamics in Ref. [28], performed below the bulk A-N transition temperature, showed that the resulting integrated form Eq. (22) very well fits experimental data. Performing our own fit of Eq. (22) to the data reported in Ref. [28] yields the values $v_{\infty}=2.59 \mu \mathrm{m} / \mathrm{s}$ and $R_{c}=42.6 \mu \mathrm{m}$, to be contrasted with results discussed below for overheated films. 


\section{Numerical results}

Figure 1 illustrates the dependence of the free energy $\Delta F$ on radius $R$ due to a growing dislocation loop in a 6 -layer film, for the case of $f(L)$ shown in Fig. 1 and $\Delta P=0$. The in-plane distance is expressed in units of $R_{s c} \equiv d \sqrt{D d / C}$, as in Fig. 2, while the free energy is plotted in units of $D d^{2}$, which follows by scaling of Eq. (18). The crosses are obtained by monitoring the numerical solution of Eq. (16) for a dynamically stable kink as it evolves with time, where the loop radius $R$ is defined such that $L(R)=[L(0)+L(\infty)] / 2$. It is seen that $\Delta F(R)$ is very well fit by Eq. (27): the fitted value of $f\left(L_{2}\right)-f\left(L_{1}\right)$ agrees with that obtained directly from the static theory with a precision of 0.5 per cent. We also verified that the right-hand-side of Eq. 200 remains constant within the same precision, giving a value $v_{\infty}=0.18 D /\left(\eta R_{s c}\right)$. This numerical calculation supports the ansatz Eq. (19) for a two-dimensional kink with constant $v_{\infty}$ related to $R(t)$ by Eq. (20). Analogous fitting to Eqs. (27) and (20) of the numerically determined $\Delta F(R)$ and kink radius has been carried out for all our calculations, showing comparable precision except very close to the maximum temperature of an $N$-layer film, where uniform thinning occurs. As a further check, we find very close agreement between the values of the line tension $E$ obtained from the fitting to Eq. (27) and by direct evaluation of Eq. (25) from the numerically determined kink profiles.

Only recently, in Ref. [13], have the dynamics of dislocation loops in overheated films been studied experimentally, albeit for a system exhibiting a first-order bulk A-isotropic (I) transition. (This difference will be discussed in Sec. V.) The magnitude of the loop velocity was found to be $10^{3}-10^{4}$ times larger than that reported for the smectic-A phase in Ref. [28], which we will comment on further below. We note that the data in Ref. [13] show a purely linear dependence of $R$ on time, suggesting that $R / R_{c} \gg 1$ in the experimentally accessible range and that the measured velocity corresponds to $v_{\infty}$ of the present theory. The data reported in Ref. [13] also show that the dislocation-loop velocity slightly increases with increasing number of layers. To check the dependence of $v_{\infty}$ on thickness in our theory we took $\eta$ to be independent of $N$. Figure 5 shows the results of calculations using the $f(L)$ function employed in Fig. 1. It is seen that in some range of thickness the velocity slightly increases with $N$ provided that $\Delta P>0$. We do not rule out that $\eta$ depends on thickness and diminishes with $N$, as suggested in Ref. [13], but a detailed analysis of $\eta$ is beyond the scope of this paper. Figure 6 shows, in the case of a 5 -layer film, that $v_{\infty}$ is predicted to increase with temperature, in agreement with experiment [13]. The rate of increase is enhanced in the presence of a nonzero $\Delta P$. The upper temperature limits of the curves in Fig. 6 are slightly less than the maximum temperatures for the given values of $N$ and $\Delta P$. Beyond these limits, dislocation-loop growth is superceded by uniform thinning.

Using Eq. (21) and the velocity data in Fig. 司, the critical radius $R_{c}$ can be determined. Referring only to the points for $N=11$ in the figure, we find that $R_{c}=14.9 R_{s c}$ and $1.43 R_{s c}$ for $\Delta P=0$ and $\Delta P=0.05 C / d^{2}$, respectively. These values bracket the range of $R_{c}$ values obtained for other values of $N$. Using the estimate for $R_{s c}$ described at the end of Sec. III, we thus find $R_{c}$ to be in the range $10^{-1}$ to $10^{-2} \mu \mathrm{m}$, several orders of magnitude smaller than the value deduced above in the bulk A phase. In view of Eq. (21), these results are consistent with the reported differences in loop velocity $v_{\infty}$ below and above the bulk transition temperature.

The key quantity which determines whether spontaneous nucleation of a dislocation loop in an overheated film actually occurs is the activation free energy $F_{a c t}$. The dependence of $F_{a c t}$ (in units of $D d^{2}$ ), for a single-layer dislocation loop, on the number of layers $N$ in the initial uniform film is shown in Fig. 7. Again, the calculations were done using the $f(L)$ function depicted in Fig. 1. Note that the temperature in this case is slightly below the maximum temperature for an 11-layer film. To judge whether nucleation occurs, we use the argument described in Ref. 12], based on the frequency per unit area of forming a dislocation loop of radius $R>R_{c}$. This frequency is given by $f=f_{0} \exp \left(-F_{\text {act }} / k_{B} T\right)$, where $f_{0}$ is estimated to be $10^{26} \mathrm{~cm}^{-2} \mathrm{~s}^{-1}$ 22. This yields [12] that the condition for a dislocation loop to nucleate in $0.1 \mathrm{~s}$ in a $1 \mathrm{~cm}^{2}$ film is $F_{a c t} / k_{B} T \approx 60$. To compare this number with Fig. 7 requires a value for the dimensionless parameter $D d^{2} /\left(k_{B} T\right)$. Taking $D=\gamma / 2 \approx 1.5 \times 10^{-2} \mathrm{~N} / \mathrm{m}, k_{B} T=4.5 \times 10^{-21} \mathrm{~J}$ and $d=3 \times 10^{-9} \mathrm{~m}$, we estimate that $D d^{2} / k_{B} T \approx 30$. These numbers yield the criterion that $F_{a c t} /\left(D d^{2}\right)$ must become about 2 or smaller for spontaneous nucleation to occur. Figure 7 shows that, for the chosen set of parameters, this can be achieved provided that $\Delta P>0$ is sufficiently large.

Figure 8 shows the variation of the line tension $E$ with initial number of layers $N$ for the same cases shown in Figs. 5 and 1 . Except for the last two points in the figure $(N=10,11), E$ is seen to be essentially independent of $\Delta P$. It follows from Eq. (28) that it is the decreasing magnitude of $E$ on approaching $N_{c r}$ for a given $T$ which is mainly responsible for the decrease in $F_{a c t}$ in Fig. 7 near $N_{c r}$ and, similarly, for the increase of $v_{\infty}$ when $\Delta P>0$ in Fig. 5. The difference between the two cases, $\Delta P=0$ and $\Delta P>0$, in Figs. 5 and 7 is due to the fact that $\tilde{f}\left(L_{2}\right)-\tilde{f}\left(L_{1}\right) \approx f\left(L_{2}\right)-f\left(L_{1}\right)+\Delta P d$ is dominated by the term $\Delta P d$ for the value of the latter used. 


\section{CONCLUSIONS}

The present theory, a modification of de Gennes' 15 theory of presmectic films, is based on the generally accepted view [12] that the occurrence of overheated free-standing films is due to surface-enhanced smectic ordering [7]. In the case of a uniform planar film, our theory 11 predicts that there is a maximum temperature for which smectic ordering in an $N$-layer film can occur. We associate this with an upper bound for the true layer-thinning transition temperature $T_{c}(N)$. Employing a dynamical generalization of the theory based on a time-dependent Ginzburg-Landau (TDGL) equation, we have shown that thinning via nucleation of dislocation loops, the mechanism indicated by recent experiments [12 14], is possible provided the pressure difference $\Delta P$ resulting from curvature of the surrounding meniscus is sufficiently large. Otherwise, the film would undergo either "uniform thinning" or, possibly, rupturing by a process analogous to spinodal dewetting [32]. The requirement for a non-zero $\Delta P$ to promote nucleation of dislocation loops is consistent with other recent studies [10,14], although we emphasize here that the condition $\Delta P \neq 0$ is not essential for some type of thinning process to occur.

In the present work, all nucleation properties (i.e., $R_{c}, F_{a c t}, v_{\infty}, E$ ) are interrelated within the framework of solutions to the TDGL equation. In particular, the line tension $E$ of the dislocation loop is expressed in terms of the kink profile, see Eq. (25). From this equation, $E$ depends implicitly on the elastic behavior of the system through the dependence of the kink shape on the uniform film free energy $\tilde{f}(L)$. This self-consistent method for incorporating the line tension differs from those used in Refs. [10,12, 14, 28].

It is important to emphasize that the present work is restricted to smectic-A liquids undergoing continuous A-N transitions in bulk. For this reason we do not attempt to make quantitative comparisons with the experimental results of Ref. [13] for dislocation loop dynamics, since the latter pertain to a system with a first-order A-I transition. Such a system can be treated using the present Landau-de Gennes theory (albeit with considerably greater complexity [20]) by appropriately modifying the bulk free-energy density in Eq. (2). One significant difference which is expected concerns contributions to the " $\Delta P$ " term in the resulting uniform-film free energy. In a system with a first-order bulk transition, such a term arises even in the absence of meniscus effects, due to the grand-canonical [17] free-energy difference between a metastable bulk smectic-A phase and the isotropic phase [9, 18]. This effect was recognized in the nucleation theory of Ref. [12], although the latter work otherwise employed de Gennes' theory for a second-order bulk transition. Clearly, to compare the present dynamical predictions with experiment, it would be of interest to perform measurements on the dynamics of layer thinning in systems such as that studied in Ref. [14], which exhibit second-order bulk A-N transitions.

Our picture is that dislocation-mediated thinning of an overheated free-standing smectic film may preempt the uniform-thinning mechanism and thus, for given $N$ and fixed values of the model parameters such as $r_{s}$, occur at a lower temperature than predicted [11 by considering a purely uniform film. This is conceivable because all freestanding film states are metastable [18]. Here we have presented several qualitative results in support of this picture. An additional step would be to evaluate the shift in layer-thinning transition temperatures $T_{c}(N)$ from those predicted 111 for a uniform film, and attempt a refitting with experimental data. This is a somewhat tedious task and is left for future study 20]. Further studies will also be directed to evaluating the kinetic coefficient $\eta$ and, as already mentioned, extending the present theory to smectic films with first-order bulk transitions.

\section{ACKNOWLEDGMENTS}

This study was supported by the Natural Sciences and Engineering Research Council (Canada).

[1] T. Stoebe, P. Mach, and C. C. Huang, Phys. Rev. Lett. 73, 1384 (1994).

[2] P. M. Johnson et al., Phys. Rev. E 55, 4386 (1997).

[3] S. Pankratz, P. M. Johnson, H. T. Nguyen, and C. C. Huang, Phys. Rev. E 58, R2721 (1998).

[4] E. I. Demikhov, V. K. Dolganov, and K. P. Meletov, Phys. Rev. E 52, R1285 (1995).

[5] E. A. L. Mol et al., Physica B 248, 191 (1998).

[6] P. Cluzeau et al., Phys. Rev. E 62, R5899 (2000). This work describes observations of layer-thinning transitions in a smectic-C liquid crystal.

[7] C. Bahr, Int. J. Mod. Phys. 8, 3051 (1994).

[8] L. V. Mirantsev, Liq. Cryst. 20, 417 (1996). 
[9] Y. Martinez-Raton, A. M. Somoza, L. Mederos, and D. E. Sullivan, Faraday Discuss. 104, 111 (1996); Phys. Rev. E 55, 2030 (1997).

[10] E. E. Gorodetskii, E. S. Pikina, and V. E. Podnek, JETP 88, 35 (1999).

[11] A. N. Shalaginov and D. E. Sullivan, Phys. Rev. E 63, 031704 (2001).

[12] S. Pankratz, P. M. Johnson, R. Holyst, and C. C. Huang, Phys. Rev. E 60, R2456 (1999).

[13] S. Pankratz, P. M. Johnson, A. Paulson, and C. C. Huang, Phys. Rev. E 61, 6689 (2000).

[14] F. Picano, P. Oswald, and E. Kats, Phys. Rev. E 63, 021705 (2001).

[15] P. G. de Gennes, Langmuir 6, 1448 (1990).

[16] D. E. Sullivan and M. M. Telo da Gama, in Fluid Interfacial Phenomena, C. A. Croxton, ed. (Wiley, 1986) p.45; M. Schick, in Les Houches, Session XLVIII, 1988 - Liquides aux interfaces, J. Charvolin, J. F. Joanny and J. Zinn-Justin, eds. (Elsevier, 1990) p.418.

[17] As appropriate for an open system, the film free energy in Eq. (2) is really the excess grand canonical potential per unit area: see [18]. Note that Eq. (2) only involves the effects of smectic ordering, and omits the contribution to the free energy of a background isotropic density variation across the film, which is assumed to be constant.

[18] Y. Martinez, A. M. Somoza, L. Mederos, and D. E. Sullivan, Phys. Rev. E 53, 2466 (1996).

[19] P. M. Chaikin and T. C. Lubensky, Principles of Condensed Matter Physics (Cambridge University Press, Cambridge, 1995).

[20] A. N. Shalaginov and D. E. Sullivan (to be published).

[21] L. Moreau, P. Richetti, and P. Barois, Phys. Rev. Lett. 73, 3556 (1994).

[22] P. G. de Gennes and J. Prost, The Physics of Liquid Crystals (Clarendon Press, Oxford, 1993); J. Prost, Adv. Phys. 33, 1 (1984).

[23] M. E. Fisher, in Statistical Mechanics of Membranes and Surfaces, D. Nelson, T. Piran and S. Weinberg, eds. (World Scientific, Singapore, 1989) p.19.

[24] B. V. Toshev and I. B. Ivanov, Col. Pol. Sci. 253, 558 (1975).

[25] P. Pieranski et al., Physica A 194, 364 (1993).

[26] S. A. Safran, Statistical Thermodynamics of Surfaces, Interfaces, and Membranes (Addison-Wesley, Reading, 1994), Ch.4.

[27] L. M. Pismen and Y. Pomeau, Phys. Rev. E 62, 2480 (2000).

[28] J. C. Geminard, R. Holyst, and P. Oswald, Phys. Rev. Lett. 78, 1924 (1997).

[29] J. A. de Feijter, in Thin Liquid Films, Fundamentals and Applications, I. B. Ivanov, ed (Dekker, New York, 1988$)$, p.1.

[30] F. Picano, R. Holyst, and P. Oswald, Phys. Rev. E 62, 3747 (2000).

[31] A. J. Bray, Adv. Phys. 43, 357 (1994).

[32] The solution of Eq. 12) at the maximum temperature when $\Delta P=0$ differs from that for $\Delta P>0$. Since $\partial \tilde{f} / \partial L=0$ at the initial film thickness $L_{0}$, there is no uniform non-stationary solution of Eq. (12) when $\Delta P=0$. Numerically-determined solutions for moving kinks (Sec. IVB) in this case exhibit a divergent kink width. A related effect is a long-range decay of the meniscus profile obtained from Eq. (7). Fluctuations omitted here are probably important in this situation, e.g., leading to film rupture or thinning by spinodal decomposition.

[33] W. van Saarloos, Phys. Rev. A 37, 211 (1988).

[34] M. Buttiker and H. Thomas, Phys. Rev. A 37, 235 (1988).

[35] V. Popa-Nita and T. J. Sluckin, J. Phys. (Paris) II 6, 873 (1996).

[36] A. N. Shalaginov, L. D. Hazelwood, and T. J. Sluckin, Phys. Rev. E 60, 4199 (1999).

[37] S. Chan, J. Chem. Phys. 67, 5755 (1977).

[38] Note that in other works [1],12,14], the quantity $\tilde{f}\left(L_{2}\right)-\tilde{f}\left(L_{1}\right)$ has been approximated from the outset by $\Delta P d$. This should be valid well in the smectic-A phase, where the minima of $f(L)$ are expected to be nearly equal, but is not generally true in the overheated regime. 


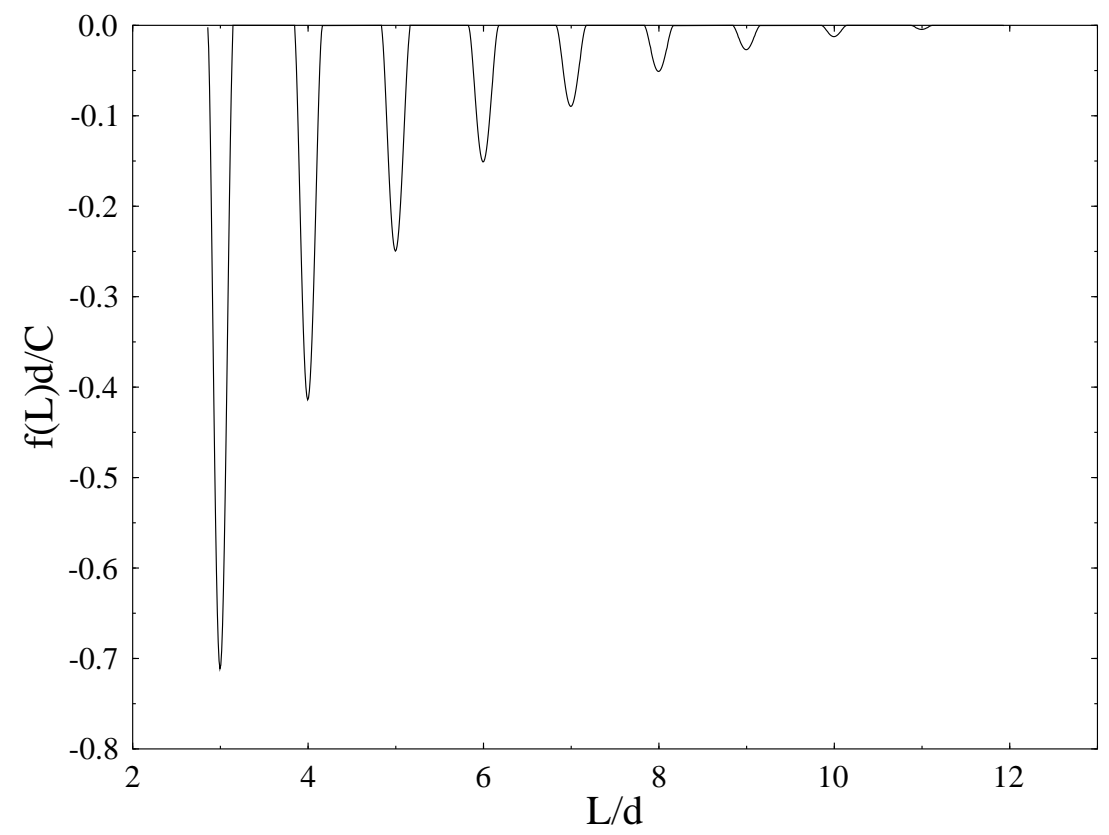

FIG. 1. The dimensionless free energy per unit area $f d / C$ vs. thickness $L / d$ calculated using scaled parameters $h_{s}=0$, $r_{s} d / C=-0.2, g d^{2} / C=0.01$ and $r d^{2} / C=0.05$.

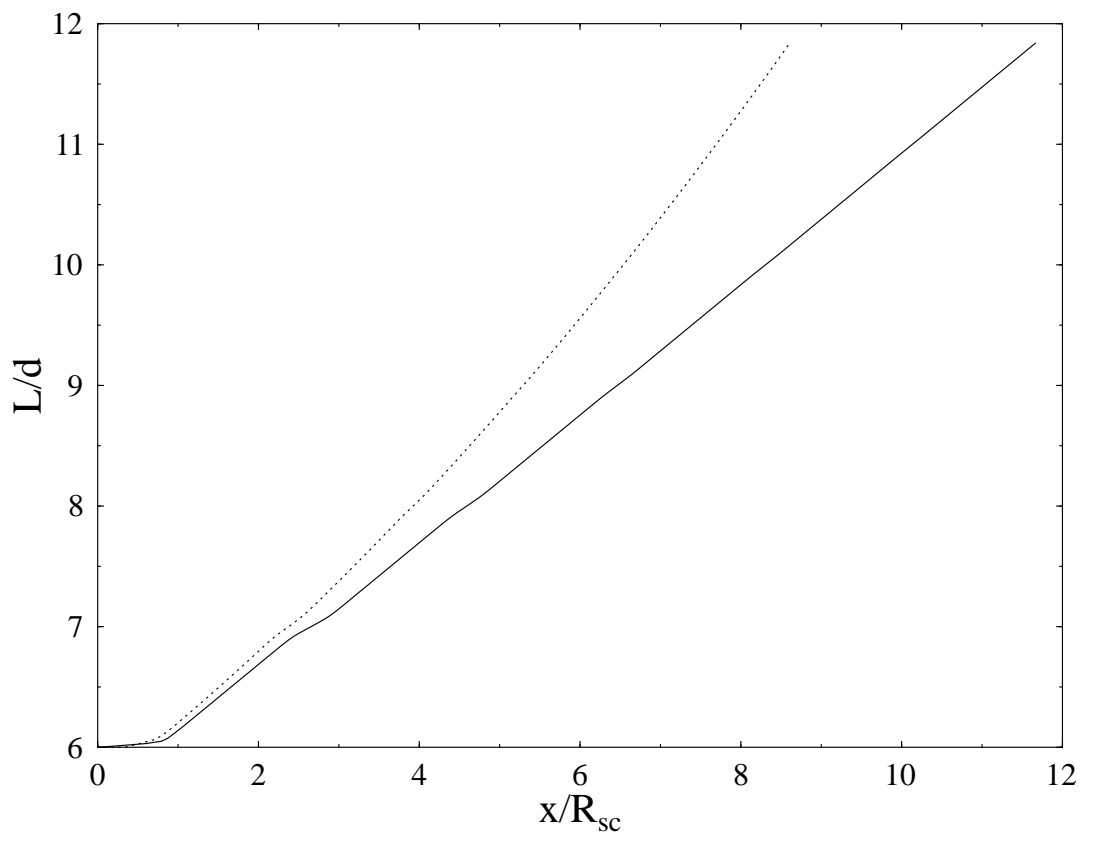


FIG. 2. Thickness of the meniscus $v s$. scaled in-plane distance $x / R_{s c}$, using the $f(L)$ function of Fig. 11 The solid line corresponds to $\Delta P=0$ while the dotted line corresponds to $\Delta P=0.05 C / d^{2}$. The origin $x=0$ is such that, for both curves, $L(x=0)=6.00001$

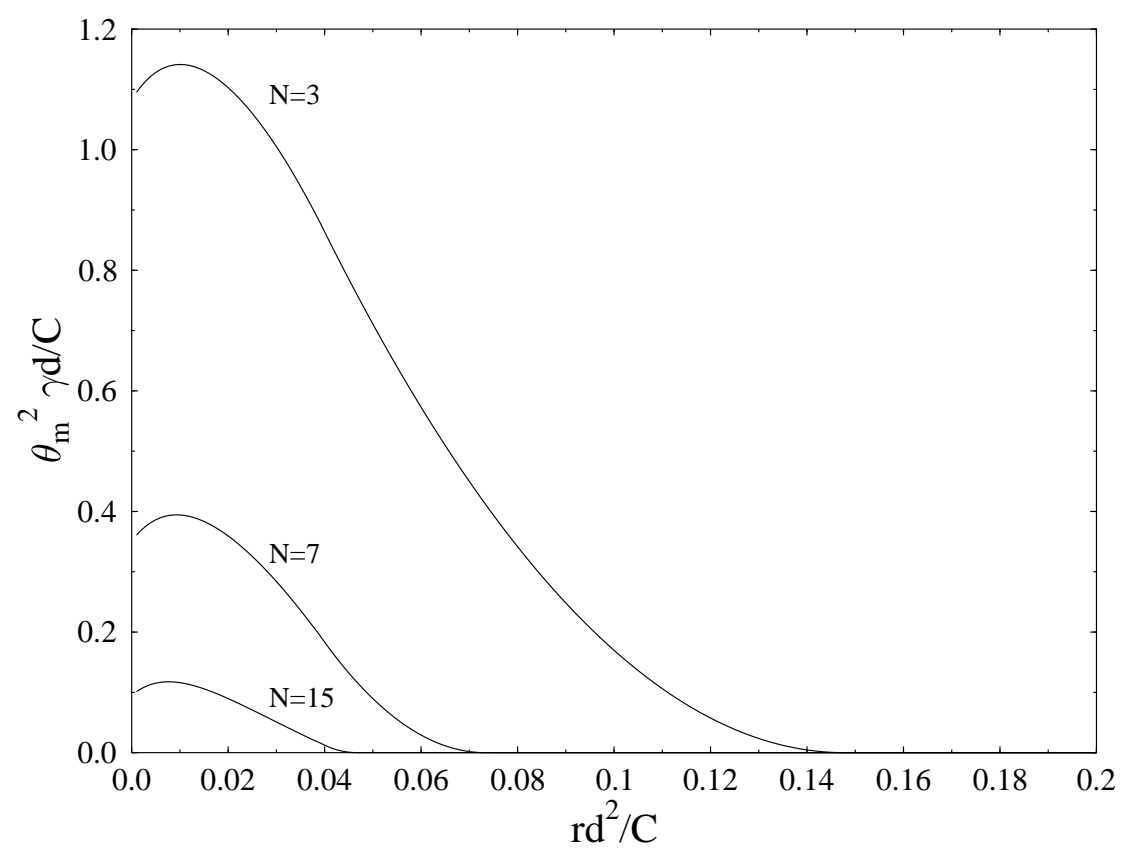

FIG. 3. Squared menisus contact angle $\theta_{m}^{2}$ in units of $C / \gamma d$ as a function of dimensionless temperature variable $r d^{2} / C \propto\left(T-T^{*}\right)$ for various number of layers $N$, calculated in the $r_{s}$-model with the same parameters as in Fig. 1. 


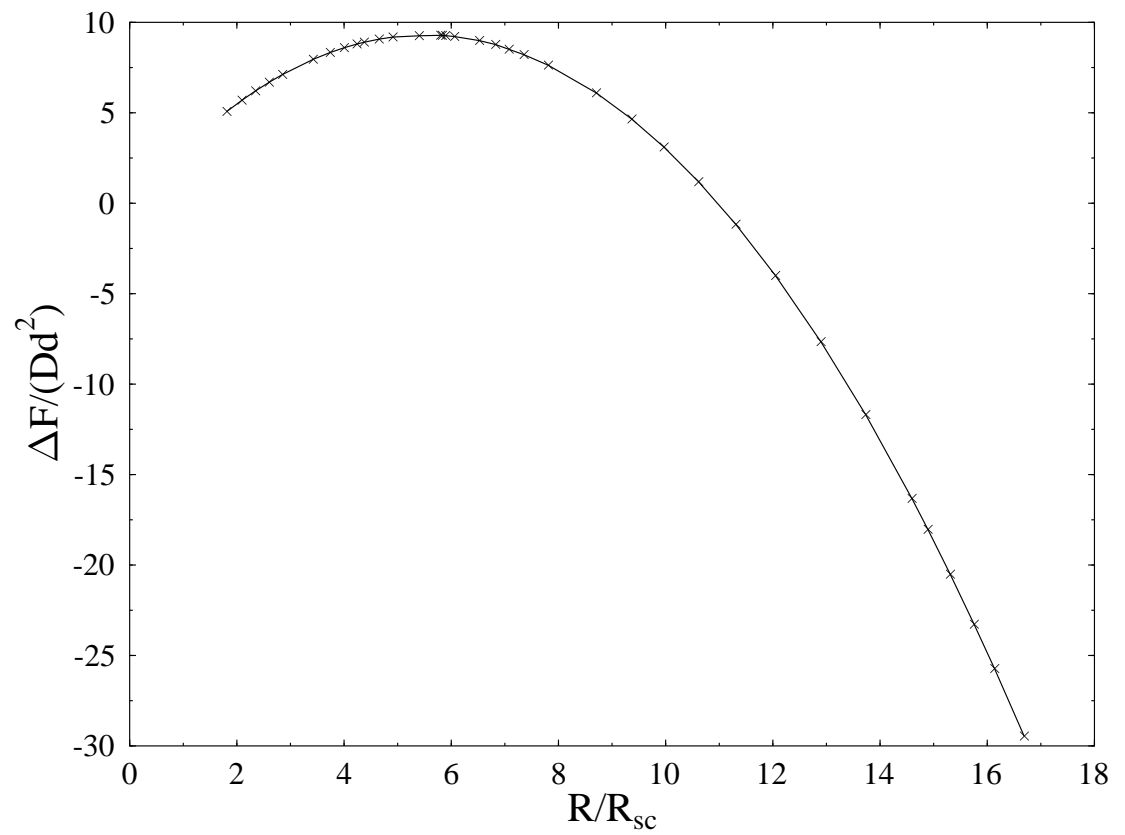

FIG. 4. Free energy $\Delta F$ (reduced by $D d^{2}$ ) of a dislocation loop in a 6-layer film vs. radius $R / R_{s c}$ Crosses: numerical solution of the dynamical equation Eq. (16) for the same $f(L)$ and $r_{s}$-model parameters as in Fig. 11, with $\Delta P=0$. Solid line: fit of $\Delta F$ with the parabolic function in Eq. 27). The best fit yields $E=0.54 \sqrt{D C d}$ and $\tilde{f}\left(L_{2}\right)-f\left(L_{1}\right)=0.098 C / d$.

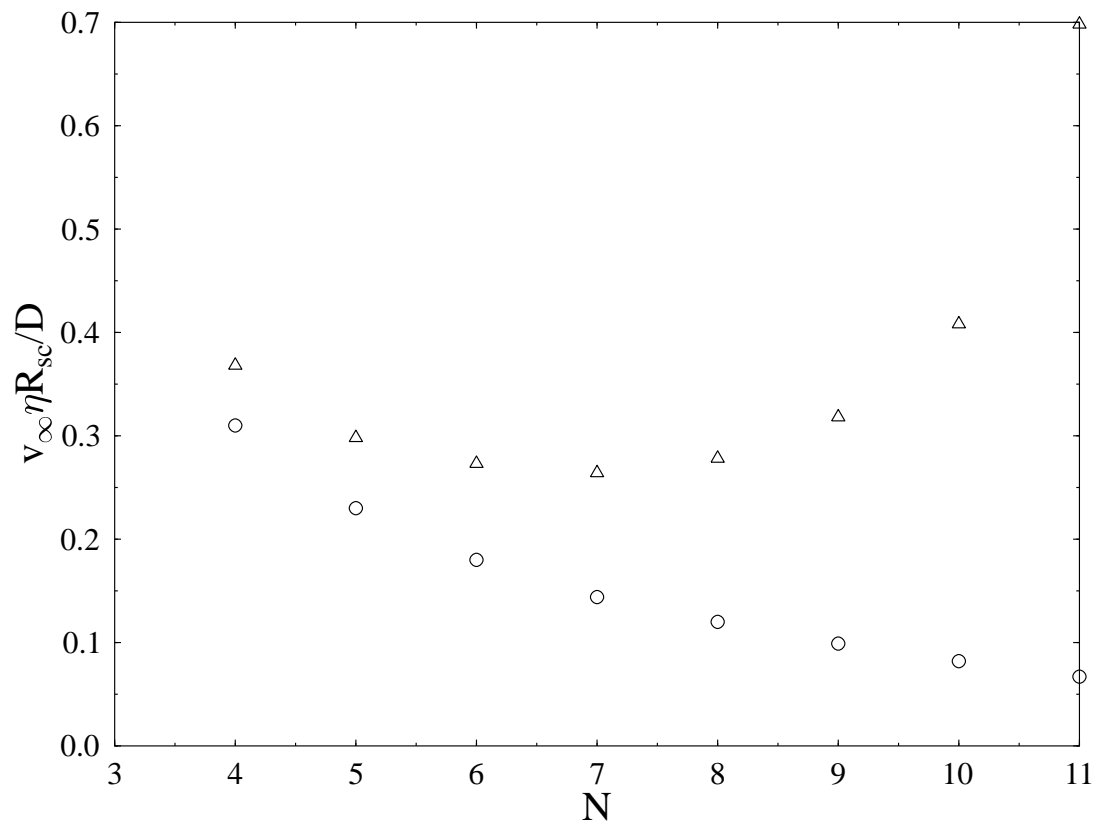


FIG. 5. Velocity $v_{\infty}$ (reduced by $D /\left(\eta R_{s c}\right)$ ) for $N$-layer films $v s$. the number of layers, calculated using the $f(L)$ depicted in Fig. 1. Circles: $\Delta P=0$. Triangles: $\Delta P=0.05 C / d^{2}$ (as in Fig. 2).

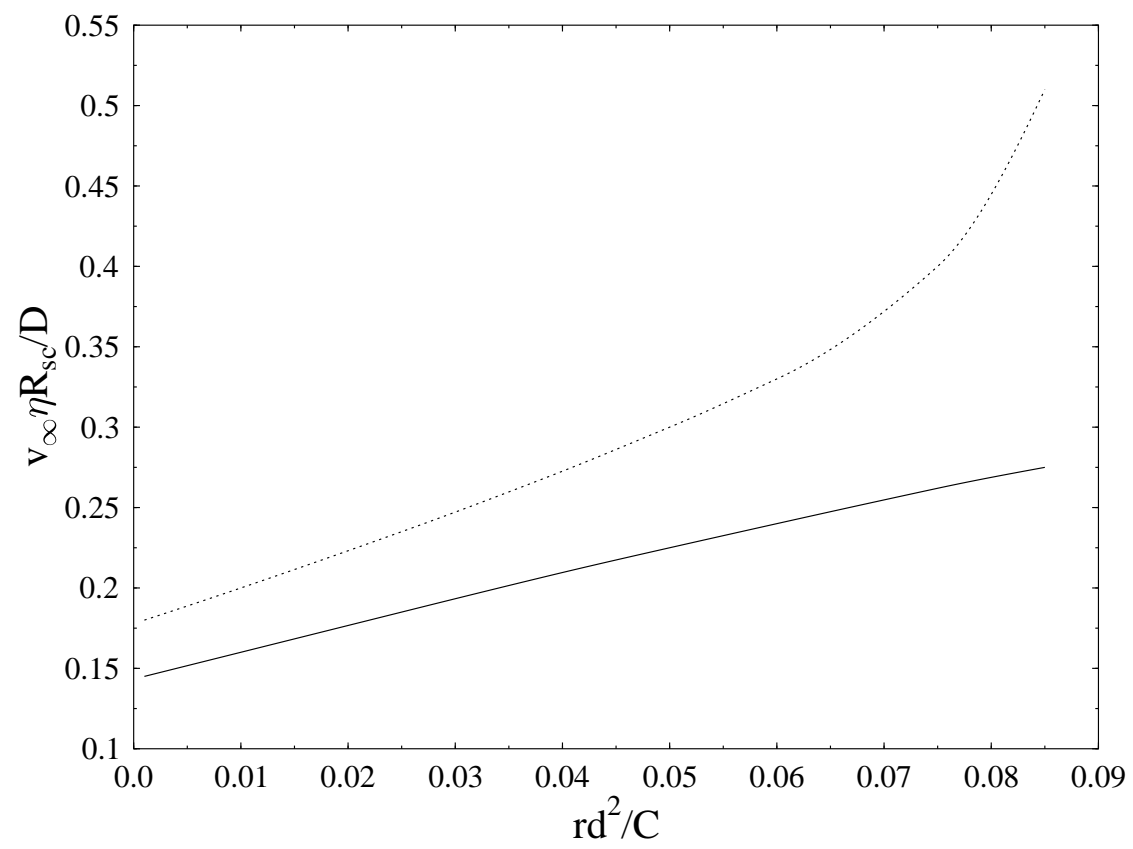

FIG. 6. Reduced velocity for a 5-layer film vs. $r d^{2} / C \propto\left(T-T^{*}\right)$. Parameters of the $r_{s}$-model are the same as in Fig. 11. Lower curve (solid): $\Delta P=0$. Upper curve (dashed): $\Delta P=0.05 C / d^{2}$. 


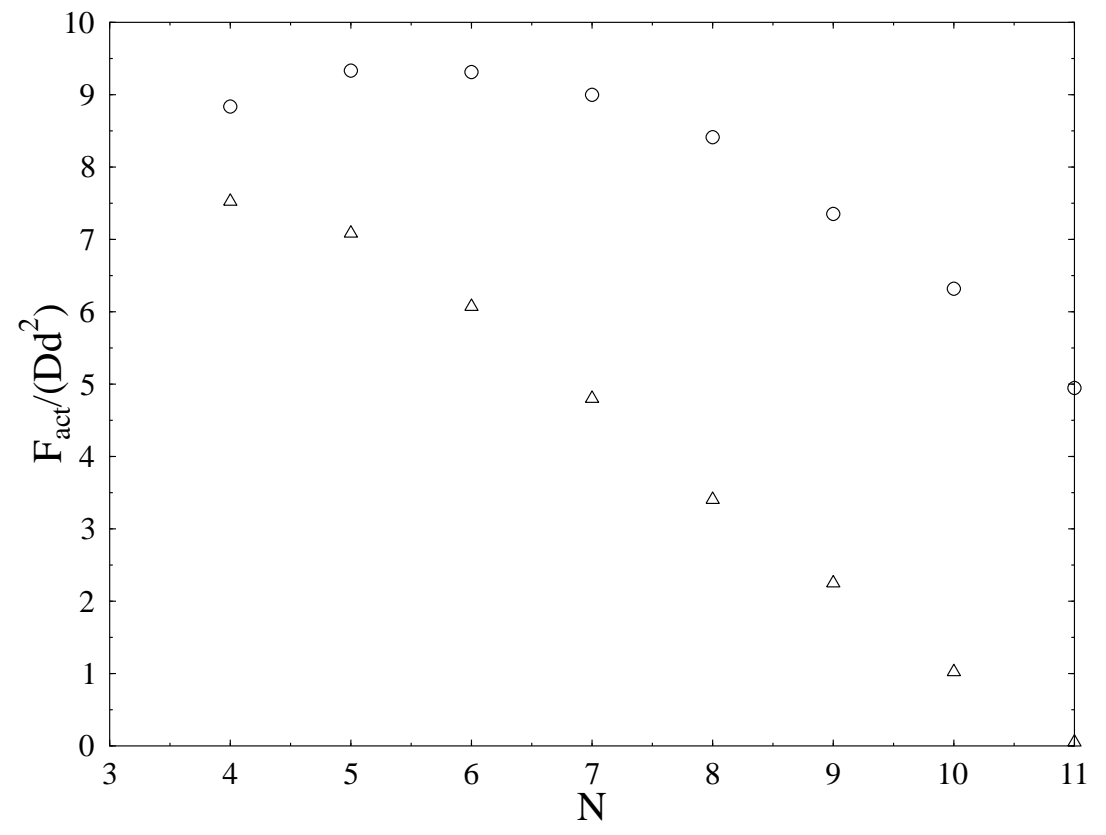

FIG. 7. Activation free energy $F_{\text {act }}$ (reduced by $D d^{2}$ ) for $N$-layer films vs. the number of layers calculated using the $f(L)$ function shown in Fig. 1. Circles: $\Delta P=0$. Triangles: $\Delta P=0.05 C / d^{2}$.

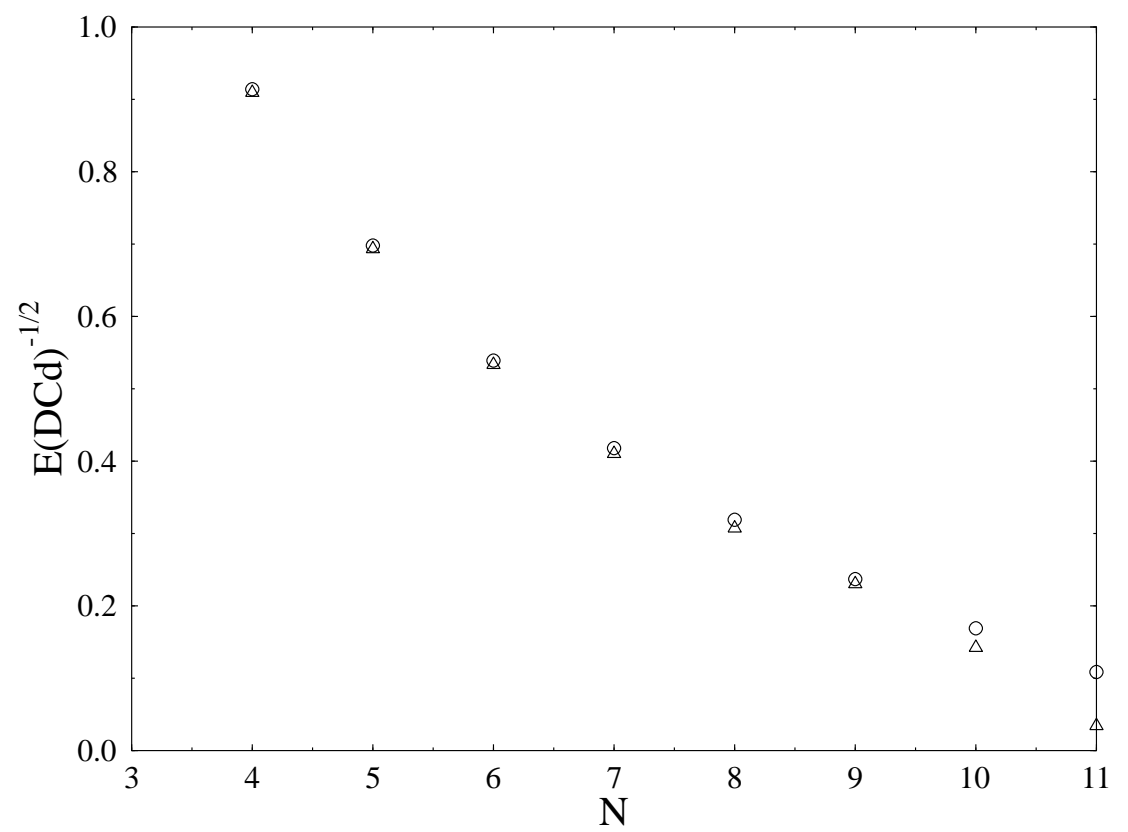


FIG. 8. Line tension $E$ (reduced by $\sqrt{D C d}$ ) for $N$-layer films $v s$. the number of layers, calculated using $f(L)$ depicted in Fig. 1. Circles: $\Delta P=0$. Triangles: $\Delta P=0.05 C / d^{2}$. 